\title{
Improved method for prediction of milled rice moisture content based on Weibull distribution
}

\author{
Jing Ling ${ }^{1,2}$, Zhaosheng Teng ${ }^{2 *}$, Haijun Lin ${ }^{3}$ \\ (1. School of Physics and Electronic-Electrical Engineering, Ningxia University, Yinchuan 750021, China; \\ 2. College of Electrical and Information Engineering, Hunan University, Changsha 410082, China; \\ 3. College of Polytechnic, Hunan Normal University, Changsha 410081, China)
}

\begin{abstract}
The loss on drying method, which is regarded as the standard method of rice moisture content analysis, provides the most reliable results but is both labor intensive and time consuming. In order to improve the detection efficiency of the loss on drying method, this study investigated the drying characteristics of milled rice and developed an information fusion algorithm with which to predict milled rice moisture content based on the Weibull distribution and Levenberg-Marquardt (LM) algorithm. Application of the Weibull distribution model was investigated regarding its description of the drying kinetics of milled rice during infrared drying. An adaptive mechanism was applied to algorithm design, with the starting point of the estimation algorithm determined by calculating the drying rate at each measuring point, and the end-point distinguished using a two-level threshold algorithm. The calculated results were then compared with the measured data regarding the infrared drying of milled rice. For milled rice samples varying in moisture content from $14.44 \%-17.67 \%$ (dry basis), the relative error between predicted and observed values ranged $0.0037-0.0589$, with a reduction in test time of 50.71\%-67.87\%.
\end{abstract}

Keywords: milled rice moisture content, Weibull distribution, Levenberg-Marquardt (LM) algorithm, information fusion, loss on drying method

DOI: $10.25165 /$ j.ijabe.20181103.3429

Citation: Ling J, Teng Z S, Lin H J. Improved method for prediction of milled rice moisture content based on Weibull distribution. Int J Agric \& Biol Eng, 2018; 11(3): 159-165.

\section{Introduction}

Rice, in the marketplace, is in different forms depending on its level of processing. After the removal of the bran layer and embryo during milling, brown rice becomes whiter in color and is known as white rice (milled rice), which is the favored form for human consumption in most countries ${ }^{[1]}$. Moisture content is one of the most important factors affecting the quality of milled rice during storage. At time of harvest, rice moisture content is high and must be reduced to nearly $14.5 \%$ (w.b. $)^{[2]}$. The need to measure and control the moisture content of milled rice is now recognized as a necessary requirement in order to maintain a competitive advantage in the food processing industry.

Several methods and systems based on newly developed technology have been proposed and applied to detect the moisture content of grain and cereals. A comparison of some of these methods is presented in Table 1 .

The loss on drying method is a reference method of moisture content measurement that is used for calibration between different species. For agricultural products, the loss on drying method has been prescribed for official inspection purposes according to the international standards AOAC $945.15-1990^{[3]}$ and ISO $712-1998^{[4]}$. Indirect methods are based on the determination of specific

\section{Receive date: 2017-04-14 Accepted date: 2018-03-07}

Biographies: Jing Ling, $\mathrm{PhD}$ candidate, Assistant Professor, research interests: intelligent detection and information fusion, Email: lingjing0807@yeah.net; Haijun Lin, PhD, Professor, research interests: intelligent detection and information fusion system, Email: linhaijun801028@126.com.

*Corresponding author: Zhaosheng Teng, PhD, Professor, research interests: intelligent detection and intelligent control system. College of Electrical and Information Engineering, Hunan University, Changsha 410082, China. Tel: +86-731- 88821111, Email: tengzs@126.com. properties of materials that depend on the sample's water content, such as electrical properties and thermal conductivity ${ }^{[5]}$.

Near infrared (NIR) spectroscopy and nuclear magnetic resonance (NMR) spectroscopy are accurate methods used to detect the chemical composition of milled rice, although the high cost and complexity of infrared spectrometric analyzers limits their applicability compared to the loss on drying method ${ }^{[6,7]}$. All of the above indirect methods, although much faster than standard techniques and easy to apply, are less accurate and must be calibrated by the standard method, typically the Karl Fischer titration method or loss on drying method.

Although the loss on drying method provides the most reliable results, it is usually labor intensive and time consuming, and therefore improving the detection efficiency of the method has become an important research subject.

Generally, there are two ways to solve this problem, the first is involving the enhancement of the heating efficiency and acceleration of the drying rate, and the second is the use of intelligent information processing to forecast the measurement results ${ }^{[8]}$. Enhancement of heating efficiency can be achieved via efficient drying techniques, such as infrared and microwave drying, with a shorter drying time, improved final dried product quality and greater energy savings considered the most important advantages of infrared drying over conventional methods ${ }^{[9]}$. Microwave drying methods are currently being explored for application in a number of fields, although not all materials can be heated rapidly by microwaves due to the occurrence of carbonization and burst phenomena during the drying process ${ }^{[10]}$. The combination of loss on drying and the predictive fusion algorithm can improve the efficiency of moisture detection by improving the weight loss under the premise of ensuring the accuracy of measurement. The predictive fusion algorithm involves the establishment of a 
mathematical model that describes the mass loss of the sample, with data processing employed to estimate moisture content when the sample is losing water but not completely dry. Recent developments in intelligent information fusion have opened up novel possibilities for moisture content detection. For example, Shrivastav and Kumbhar ${ }^{[11]}$ studied the superheated steam drying characteristics of paneer, proposing an optimized ANN model with two hidden layers to predict the moisture content of the sample. Elsewhere, Ling et al. ${ }^{[12]}$ proposed a semi-empirical model for the prediction of pork moisture content based on Fick's second law, as well as a prediction and fusion algorithm for meat moisture content detection during loss-on-drying using the least squares method.

Recently, many studies covered the mathematical modeling and kinetics of the drying process by several researchers. Each of these empirical and semi-empirical models describe the impact of drying conditions on the drying process to varying degrees, since drying kinetics are greatly affected by drying conditions. Among the empirical models, the Weibull distribution has been used to describe the behavior of drying kinetics for different drying variables $^{[13]}$. However, little information is available regarding the use of the Weibull model for the detection of grain and cereal moisture content based on the loss on drying method.

The objectives of this study were: (1) to determine the applicability of the Weibull model in predicting the moisture content of milled rice during infrared drying; (2) to develop and realize a prediction and fusion algorithm for milled rice moisture content detection during drying, using the LM algorithm; and (3) to evaluate the feasibility of the fusion algorithm for milled rice moisture content detection. This study focuses on the actual applicability of the algorithm and modeling to illustrate the drying kinetics of milled rice, with the research results obtained potentially beneficial in finding an effective method suitable for small embedded systems used in the real-time signal processing of predictive moisture analyzers.

Table 1 Comparison of methods used to determine moisture content

\begin{tabular}{|c|c|c|c|}
\hline \multicolumn{2}{|c|}{ Detection method } & Advantages & Disadvantages \\
\hline $\begin{array}{l}\text { Loss on drying } \\
\text { method }\end{array}$ & $\begin{array}{l}\text { Electric drying method } \\
\text { Infrared drying method } \\
\text { Microwave drying }\end{array}$ & $\begin{array}{c}\text { Accurate, extensive applicability, well established } \\
\text { and does not demand sophisticated laboratory } \\
\text { equipment }{ }^{[4]}\end{array}$ & Labor intensive and time consuming \\
\hline \multirow{2}{*}{ Indirect methods } & $\begin{array}{l}\text { Resistance method } \\
\text { Capacitance method }\end{array}$ & $\begin{array}{c}\text { Low cost of apparatus, rapid non-destructive } \\
\text { determination }\end{array}$ & $\begin{array}{l}\text { Results are easily influenced by quality factors (varieties, moisture } \\
\text { content, aging degree, crack rate, grain type environment) and } \\
\text { storage conditions (storage time, bulk density); poor repeatability }{ }^{[6]}\end{array}$ \\
\hline & $\begin{array}{l}\text { NIR spectroscopy } \\
\text { NMR spectroscopy }\end{array}$ & Accurate, rapid non-destructive determination & $\begin{array}{l}\text { High cost and complexity of apparatus; results are easily influenced } \\
\text { by measuring conditions (environmental temperature, humidity, } \\
\text { storage time and bulk density) }{ }^{[7]}\end{array}$ \\
\hline
\end{tabular}

\section{Materials and methods}

\subsection{Preparation of milled rice samples}

Milled rice was purchased from the Longping Seed Industry Co. Ltd, Changsha, China, with the reference moisture content (MC) of the samples varying from $14.44 \%-17.67 \%$ (dry basis), as determined by the loss on drying method (AOAC, 2000) ${ }^{[4]}$. The milled rice samples with different $\mathrm{MC}$ values were divided into 12 equal portions, placed in sealed polyethylene bags and stored at a temperature of $5^{\circ} \mathrm{C} \pm 1^{\circ} \mathrm{C}$ to ensure no additional moisture loss prior to the drying tests. A powder was then made out of the 12 samples by grinding in a grinding mill (HONGTAI, HLO-400, China) and screening through $1.5 \mathrm{~mm}$ mesh according to the AOAC loss on drying method.

\subsection{Instruments and equipment}

In this study, a moisture analyzer (SARTORIUS, MA100, Germany) was used as the drying apparatus (Figure 1). According to the experimental set-up illustrated in Figure 1, a ceramic infrared radiator of circular shape was fixed on the underside of the top cover of the analyzer, with a sensitive thermometer measuring the temperature of the heated chamber. As the sample is heated, the water molecules evaporate, the weight of the sample decreased. A microcontroller circuit detects this change in weight over time as the sample is dried via infrared heating ${ }^{[14]}$. The technical specifications of the apparatus are as follows ${ }^{[15]}$ : Max. weighing capacity: $100 \mathrm{~g}$; accuracy of the weighing system: $0.1 \mathrm{mg}$; repeatability: $\pm 0.1 \%$ for initial sample weight approx. $>1 \mathrm{~g}$; reproducibility of the temperature: $1 \%$; power consumption: max. $700 \mathrm{VA}$; voltage frequency: 48-60 Hz; temperature range and settings: $30^{\circ} \mathrm{C}-230^{\circ} \mathrm{C}$, adjustable in $1^{\circ} \mathrm{C}$ increments.

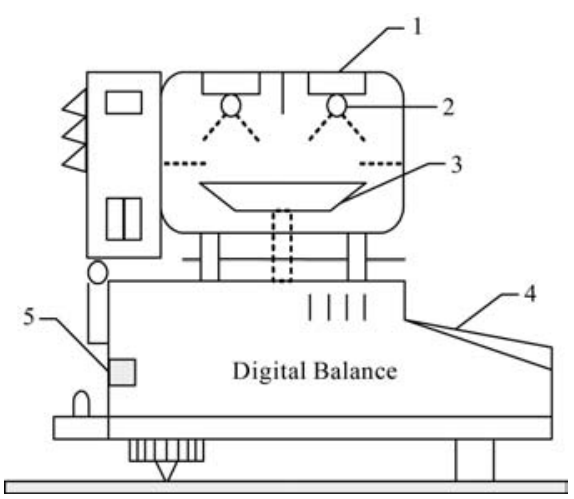

1. Temperature sensor 2. IR radiator 3. Aluminum drying tray 4. Control bar and display 5. Serial interface

Figure 1 Experimental set-up (SARTORIUS MA100 moisture analyzer)

\subsection{Experimental procedure}

The aluminum-plate drying trays $(2 \mathrm{~mm}$ in thickness and $90 \mathrm{~mm}$ in diameter) were used to hold the samples. An aluminum tray was selected as its high surface reflectivity was considered to minimize radiation energy loss. The tray was pre-dried for minimum of $1 \mathrm{~h}$ under the conditions specified, cooled in the desiccators and weighed, with this drying and weighing procedure repeated until the pre- and post-drying weight difference was not greater than $0.005 \mathrm{~g}^{[4]}$. Around 5-10 $\mathrm{g}$ of sample powder was then placed in the drying tray in a thin, even layer to a height of approximately 2-5 $\mathrm{mm}$. Drying experiments were performed at a drying temperature of $105^{\circ} \mathrm{C}$, with the entire experimental procedure following the AOAC loss on drying method. The mass of the samples were measured at $6 \mathrm{~s}$ intervals by the moisture analyzer and uploaded to the computer through the RS-232 
interface, saved in a database and retrieved by the prediction and fusion algorithm. All the above calculation procedures were programmed in Matlab 2013a (The Mathworks, Inc., Natick, MA, USA). All moisture content determinations were replicated three times.

\section{Results and discussion}

\subsection{Mathematical modeling of infrared drying of milled rice}

According to ISO $712-1998$, the standard temperature $\left(105^{\circ} \mathrm{C}\right)$ is used for moisture detection in cereals and cereal products ${ }^{[3]}$. In this method, the sample is subjected to drying at a temperature of $105^{\circ} \mathrm{C}$ for various specified times or until complete dryness.

The characteristic drying curve of the infrared drying is shown in Figure 2. This graph clearly shows that the drying rate, which is the slope of the drying curve, decreases with drying progress. During the infrared drying process water loss accelerates during the heating up period $A B$ (i.e., the second derivative of the drying curve is less than 0 ), with the maximum drying rate related to the heat transfer rate of the sample. Whereas the constant rate period $B C$ is very short (the second derivative of the drying curve is equal to 0 ), the falling rate period $C D$ lasts for some time, as the water loss rate of the sample slows progressively (the second derivative of the drying curve is greater than 0$)^{[16]}$. The purpose of predicting the milled rice moisture content is to fit the sample drying curve for period $C D$ to obtain a result with time $t$ (or a function value that meets the threshold), so as to correctly estimate moisture content when the sample is losing water but not completely dry.

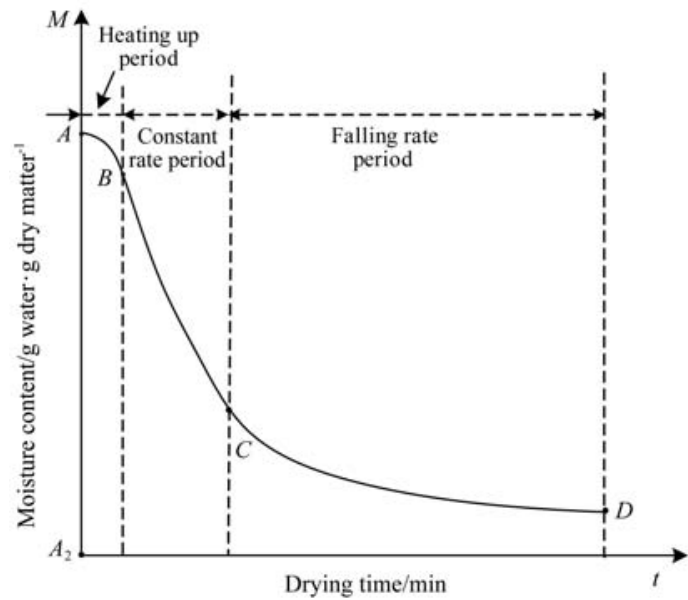

Figure 2 Characteristic drying curve of the infrared drying

The Weibull model was originally developed to represent the distribution of the breaking strength of materials and later to describe the behavior of systems or events exhibiting some degree of variability ${ }^{[17]}$. It has been widely used to analyze the kinetics of biochemistry ${ }^{[18]}$, the behavior of rehydration kinetics ${ }^{[19]}$ processes, and to describe the behavior of drying kinetics, due to its simplicity and flexibility.

The obtained infrared drying curves of milled rice were fitted with the Weibull distribution function model, in which the moisture ratio $M R$ of the milled rice during the infrared drying experiments can be expressed as:

$$
\begin{gathered}
M R=\frac{M_{t}-M_{e}}{M_{0}-M_{e}}=\exp \left[-\left(\frac{t}{\alpha}\right)^{\beta}\right] \\
M_{t}=\left(M_{0}-M_{e}\right) \exp \left[-\left(\frac{t}{\alpha}\right)^{\beta}\right]+M_{e}
\end{gathered}
$$

where, $M_{t}$ is moisture content, $\mathrm{g}$ water/g dry matter; $M_{e}$ and $M_{0}$ are the equilibrium and initial moisture content, $\mathrm{g}$ water/g dry matter; $\alpha$ is the scale parameter of the Weibull model; $\beta$ is the shape parameter; and $t$ is the sampling time, min. $\quad \alpha$ defines the rate and represents the time needed to accomplish approximately $63 \%$ of the process $^{[20]} . \quad \beta$ is related to velocity of the mass transfer at the beginning, e.g., the lower the $\beta$ value is, the faster the drying rate is at the beginning ${ }^{[21]}$.

After the models were built, twelve sets of randomly selected test data were used to evaluate their performance. In order to illustrate the curve fitting criteria of mathematical models at different moisture content, the non-regression analysis was performed using the curve fitting toolbox of MATLAB 2013a and calculate the $\alpha$ and $\beta$, as shown in Table 2. The moisture content of the milled rice is expressed as a percentage of moisture based on wet basis $\left(M_{\mathrm{wb}}\right)$ and dry basis $\left(M_{\mathrm{db}}\right)$. The coefficient of determination $\left(R^{2}\right)$ was the primary criterion used to selecting the equation best able to describe the drying curve, with reduced chi-square $\left(\chi^{2}\right)$ and root mean square error (RMSE) values used to determine the best fit.

Table 2 Curve fitting criteria of mathematical models at different moisture content values

\begin{tabular}{cccccccc}
\hline No. & $M_{\mathrm{wb}} / \%$ & $M_{\mathrm{db}} / \mathrm{g} \cdot \mathrm{g}^{-1}$ & $\beta$ & $\alpha$ & $R^{2}$ & $\chi^{2}$ & $R M S E$ \\
\hline 1 & 12.84 & 0.1473 & 0.9089 & 31.51 & 0.9903 & $2.750 \times 10^{-4}$ & 0.0163 \\
2 & 13.53 & 0.1565 & 0.9234 & 35.34 & 0.9997 & $1.871 \times 10^{-4}$ & 0.0102 \\
3 & 13.90 & 0.1614 & 0.9102 & 38.28 & 0.9692 & $3.057 \times 10^{-4}$ & 0.0325 \\
4 & 14.37 & 0.1678 & 0.9277 & 49.45 & 0.9897 & $2.077 \times 10^{-4}$ & 0.0423 \\
5 & 14.12 & 0.1644 & 0.9125 & 43.56 & 0.9697 & $4.672 \times 10^{-4}$ & 0.0752 \\
6 & 12.62 & 0.1732 & 0.9045 & 37.83 & 0.9993 & $4.410 \times 10^{-4}$ & 0.0433 \\
7 & 13.92 & 0.1619 & 0.9117 & 39.14 & 0.9912 & $3.251 \times 10^{-4}$ & 0.0684 \\
8 & 14.77 & 0.1732 & 0.9146 & 47.76 & 0.9689 & $2.051 \times 10^{-4}$ & 0.0411 \\
9 & 13.94 & 0.1619 & 0.9061 & 37.44 & 0.9932 & $5.667 \times 10^{-4}$ & 0.0128 \\
10 & 13.88 & 0.1611 & 0.9148 & 31.99 & 0.9668 & $3.789 \times 10^{-4}$ & 0.0315 \\
11 & 14.34 & 0.1674 & 0.9171 & 40.16 & 0.9996 & $2.864 \times 10^{-4}$ & 0.0093 \\
12 & 15.02 & 0.1767 & 0.9359 & 48.78 & 0.9998 & $1.449 \times 10^{-4}$ & 0.0328 \\
\hline
\end{tabular}

Model evaluations were necessary in order to estimate the accuracy and robustness of their predictive ability. According to the statistical results shown in Table 2, the $R^{2}$ of Weibull model (0.9668-0.9998), $\chi^{2}\left(1.449 \times 10^{-4}-5.667 \times 10^{-4}\right)$, and RMSE (0.00930.0752 ) could be regarded as reasonably results. The shape parameter $\beta$ varied in a narrow range from 0.9045 to 0.9359 , and the scale parameter $\alpha$ ranged from 31.51 to 48.78 (Table 2). In order to visualize the suitability of the Weibull model in describing the infrared drying of milled rice, we randomly selected a set of samples, the initial moisture content of which was 0.1659 (dry basis).

The curves of observed and predicted moisture content based on the Weibull model are described in Figure 3a and their absolute errors represented in Figure 3b, for 19 sampling points. In Figure $3 \mathrm{a}$, the fitting curve is banded around the drying curve, which demonstrated the suitability of the mathematical model, while in Figure $3 \mathrm{~b}$, the absolute error between measured and predicted values is lower than $3.0 \times 10^{-3}$.

\subsection{Estimation of model parameters}

The moisture content $M_{t}$ (d.b.) and the parameter vector $\left[M_{e}, M_{0}, \alpha, \beta\right]$ at any time $t$ satisfy the nonlinear function shown in Equation (2). The non-linear curve fitting of $M_{t}$ can be transformed into solving the nonlinear parameter optimization problem of Equation (2) based on the observations of $t$ and $M_{t}$ for the least-square significance. 


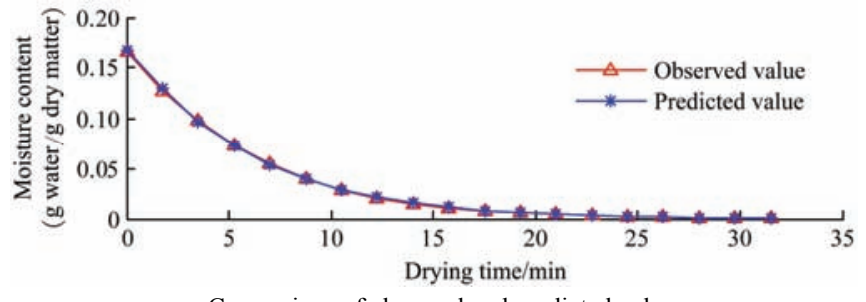

a. Comparison of observed and predicted values

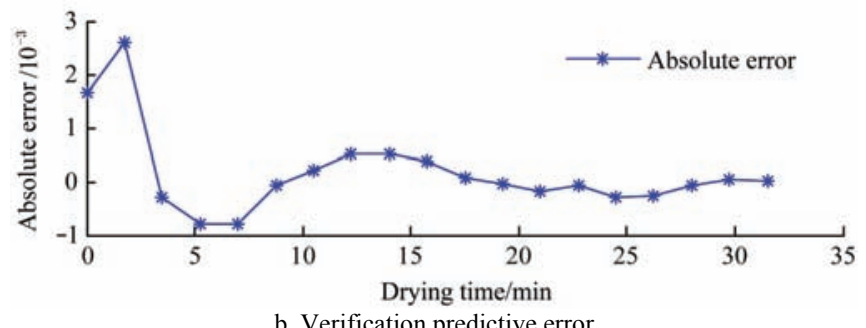

b. Verification predictive error

Figure 3 Error analysis of the predictive fusion algorithm

The parameter estimation is obtained when the minimization of the difference between calculated and measured moisture content is achieved. The Levenberg-Marquardt (LM) algorithm is a modification of the Gauss-Newton method that, in a self-adaptive manner, navigates between Gauss-Newton and the method of steepest descent ${ }^{[22]}$.

\subsubsection{LM algorithm}

Assuming functional relation $f(x, \bar{k})$ that maps a parameter vector $\bar{k}, \bar{k}=\left(k_{1}, k_{2}, k_{3}, \ldots k_{n}\right)^{T}, \bar{k} \in R^{n}$. The measured vectors $\left(x_{\mathrm{i}}, y_{\mathrm{i}}\right), i=0,1,2 \ldots, m-1$, and $m>n$ are provided, with the desired result being to find the vector $\bar{k}$ that best satisfies the functional relation $f$. The problem is thus to compute those estimates of the parameters which will minimize ${ }^{[23]}$.

$$
\left.\psi(\bar{k})=\sum_{i=1}^{m} d_{i}^{2}=\sum_{i=1}^{m}\left\{y_{i}-f\left(x_{i}, \bar{k}\right)\right]\right\}^{2}
$$

where, $y_{i}$ is the measured value; $f\left(x_{i}, \bar{k}\right)$ is the calculated value, and $m$ is the number of measurement points. The method then involves correcting the unknown set of parameters using the following formula:

$$
\bar{k}_{j+1}=\bar{k}_{j}-[H+\lambda I]^{-1} \nabla \psi\left(\bar{k}_{j}\right)
$$

where, $H=\nabla^{2} \psi(\bar{k})$ is the Hessian matrix evaluated at $\bar{k}_{j}$; $\nabla \psi(\bar{k})$ is the Jacobian matrix $\frac{\partial \psi\left(\bar{k}_{j}\right)}{\partial \bar{k}_{j}} ; I$ is the unit matrix, and $\lambda$ is the damping parameter. Marquardt later replaced the unit matrix in Equation (4) with the diagonal of the Hessian, resulting in the LM update rule. Thus, Equation (4) can be modified as:

$$
\bar{k}_{j+1}=\bar{k}_{j}-[H+\lambda \operatorname{diag}(H)]^{-1} \nabla \psi\left(\bar{k}_{j}\right)
$$

The success of this estimation procedure is associated with $\lambda$ in Equation (5), with the update rule used as follows. If the error decreases following an update, the assumption regarding $f\left(x_{i}, \bar{k}\right)$ is implied as working, and $\lambda$ decreasing (usually by a factor of 10 ) to reduce the influence of gradient descent. On the other hand, if the error increases, we would like to follow the gradient more closely and then $\lambda$ is increased by the same factor ${ }^{[24]}$.

The goal of this study is the estimation of the parameters described in Equation (2) via the LM method, the source term presented above is defined mainly by four parameters $\left[M_{0}, M_{\mathrm{e}}, \alpha\right.$, $\beta]$ :

$$
\psi=\sum_{i=i}^{m} d_{i}^{2}=\sum_{i=1}^{m}\left\{y_{i}-\left[\left(M_{0}-M_{e}\right) \exp \left[-\left(\frac{t}{\alpha}\right)^{\beta}\right]+M_{e}\right]\right\}^{2}
$$

The Jacobian matrix is written as:

$$
\nabla \psi(\bar{k})=\left[\begin{array}{cccc}
\frac{\partial \psi_{1}}{\partial M_{e}} & \frac{\partial \psi_{1}}{\partial M_{0}} & \frac{\partial \psi_{1}}{\partial \alpha} & \frac{\partial \psi_{1}}{\partial \beta} \\
\frac{\partial \psi_{2}}{\partial M_{e}} & \frac{\partial \psi_{2}}{\partial M_{0}} & \frac{\partial \psi_{2}}{\partial \alpha} & \frac{\partial \psi_{2}}{\partial \beta} \\
\mathrm{M} & \mathrm{M} & \mathrm{M} & \mathrm{M} \\
\frac{\partial \psi_{m}}{\partial M_{e}} & \frac{\partial \psi_{m}}{\partial M_{0}} & \frac{\partial \psi_{m}}{\partial \alpha} & \frac{\partial \psi_{m}}{\partial \beta}
\end{array}\right]
$$

where, the elements of the matrix are defined as the first derivative of $\psi(\bar{k})$ at position $y_{i}$ with respect to the unknown parameter $\bar{k}_{j} \in \bar{k}=\left[M_{e}, M_{0}, a, \beta\right]$, and approximated Hessian matrix $H=\nabla \psi(\bar{k})^{T} \nabla \psi(\bar{k})$.

\subsubsection{Initial values of the algorithm parameters}

In this study, the special points $t \rightarrow \infty$ and $t \rightarrow 0$ were used to calculate the initial values of the parameters. Equation (2) can thus be modified as:

$$
M_{t}=\left(M_{0}-M_{e}\right) \exp \left[-\left(\frac{t}{\alpha}\right)^{\beta}\right]+\left.M_{e}\right|_{t \rightarrow \infty}=M_{e}
$$

A value of $t \rightarrow \infty$ signifies the end of the drying process, and $M_{t} \approx M_{e}$. From the knowledge of infrared drying kinetics, the magnitude of the equilibrium moisture content $\left(M_{e}\right)$ tends to be very small ${ }^{[25]}$, so we set $M_{e}=0.01$. Conversely, a value of $t \rightarrow 0$ signifies the starting point of the drying process and $M_{t}=M_{0}$. The permitted milled rice moisture content according to the Chinese standard GB $1354-2009^{[26]}$ can be seen as a priori knowledge of the initial moisture content; thus we set $M_{0}=0.15$ (dry basis).

$$
\begin{aligned}
M_{t} & =\left(M_{0}-M_{e}\right) \exp \left[-\left(\frac{t}{\alpha}\right)^{\beta}\right]+\left.M_{e}\right|_{t=0} \\
& =\left(M_{0}-M_{e}\right)+M_{e}=M_{0}
\end{aligned}
$$

The values of the scale parameter $\alpha$ and shape parameter $\beta$ calculated in Table 2 can be substituted into the predictive fusion algorithm as prior knowledge. According to the experimental experience, we set $\alpha=30$ and $\beta=0.9$ as the initial value in this work.

\subsection{Adaptive recognition for the starting point of the predictive algorithm}

In the presented approach, the starting point of estimation (the point that separates the heating up and falling rate drying processes) is crucial. This starting point can also be defined as the inflection point, and is calculated using the second derivative of the drying curve. In contrast, the end-point criterion of the method depends on the calculation of the drying rate and the estimated moisture content. Here a two-level threshold criterion method is designed to distinguish the endpoint.

The first derivative of the sample mass denote as $v$ (rate of water loss), and the second derivative of sample mass denote as $a$ (acceleration of water loss):

$$
\begin{gathered}
v=\frac{d m_{i}}{d t}=\frac{m_{i}-m_{i+1}}{t_{i+1}-t_{i}} \\
a=\frac{d^{2} m}{d t^{2}}=\frac{\Delta^{2} m_{i}}{(\Delta t)^{2}}=\frac{\left(m_{i-1}-m_{i}\right)-\left(m_{i}-m_{i+1}\right)}{\left(\Delta t_{i}\right)^{2}}
\end{gathered}
$$

where, $m_{i}$ and $m_{i-1}$ are the mass of the sample at $i$ and $i-1, \mathrm{~g} ; t_{i}$ is the drying time, min; $\Delta m$ and $\Delta t$ are the differences in sample mass and 
time, respectively. Moisture content is usually expressed in \% of moisture present, and there are two methods for expressing these percentages: (i) wet basis (w.b.), and (ii) dry basis (d.b.):

$$
\begin{gathered}
M_{\mathrm{wb}}=\frac{m_{t}-m_{e}}{m_{0}} \times 100 \%, M_{\mathrm{db}}=\frac{M_{\mathrm{wb}}}{100-M_{\mathrm{wb}}} \\
M_{\mathrm{db}}=\frac{m_{t-} m_{e}}{m_{0}-m_{t}+m_{e}}
\end{gathered}
$$

where, $M_{\mathrm{wb}}$ is the moisture content of dry basis, \%; and $M_{\mathrm{wb}}$ is the moisture content of dry basis, $g$ water/g dry matter; $m_{t}$ is the mass of the sample at $t, \mathrm{~g} ; m_{0}$ and $m_{e}$ are the initial and final mass of the sample, g. From Equations (12) and (13), the moisture content is expressed as the ratio of the mass of the sample. The numerator and denominator in the Equation (13) are greater than zero. The variation trend of moisture content $\left(M_{\mathrm{db}}\right)$ during drying is similar to that of sample mass loss $\left(m_{t}\right)$. The inflection point of the drying curve is the same as the inflection point of the sample mass loss.

To visualize this view, milled rice samples were here randomly selected, with the initial moisture content $M_{\mathrm{db}}=14.76 \%$ (dry basis) and initial mass $m_{0}=4.991 \mathrm{~g}$. When $\mathrm{T}=105^{\circ} \mathrm{C}$, the infrared drying curve was measured and recorded at a time interval of $6 \mathrm{~s}$. The derivative of the sample mass $m_{0}$ and moisture content $M_{\mathrm{db}}$ then calculated in order to illustrate the determination of the inflection point, as shown in Figure 4.

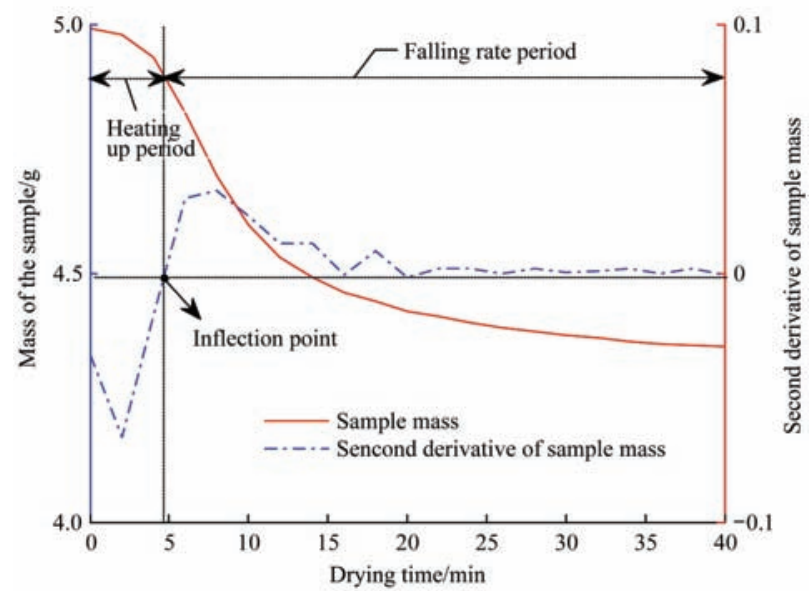

b. Variation of sample mass $m_{t}$

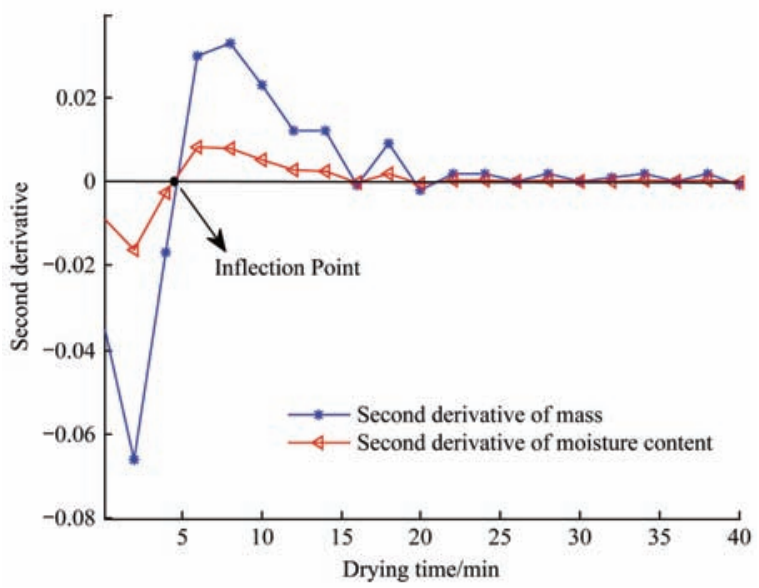

c. Second derivative of sample mass $m_{t}$ and moisture content $M_{\mathrm{db}}$

Figure 4 Determination of drying curve inflection point

The trend of moisture content is similar with the decrease of sample mass, as shown in Figures $4 \mathrm{a}$ and $4 \mathrm{~b}$. It can be seen from Figure $4 \mathrm{c}$ that the point where the second derivative of two variables equals zero is the same. Therefore, the starting point of the predictive fusion algorithm can be calculated by calculating the second derivative of the sample mass.

According to the drying curves, which are shown in Figures $4 \mathrm{a}$ and $4 \mathrm{~b}, a<0$ during the heating period and $a>0$ during the falling rate period. Thus, the starting point of prediction can be determined by judging whether $a$ is positive or negative:

$$
S= \begin{cases}1, & \text { if } a_{i}<0 \text { and } a_{i+1}>0 \\ 0, & \text { others. }\end{cases}
$$

where, $a_{i} i$ s the acceleration of water loss at $i$. If $S=1$, then $i+1$ is the starting point. However, due to experimental noise, the recorded drying curve is not smooth, thus Equation (14) needs to be modified.
If $i+1$ meets the requirement of $S=1$ and the subsequent $K$ points all meet the requirement of $a>0$, then $i+1$ is determined as the starting point of prediction. Also considering $a=0$, Equation (14) can then be modified as:

$$
S= \begin{cases}1, & \text { if } a_{i}<0 \text { and } a_{i+1} \geq 0 \text { and } a_{i+1+j} \geq 0, j=1,2, \ldots, K \\ 0, & \text { others. }\end{cases}
$$

where, $K$ can be determined via experimental analysis. The greater the value of $K$ is, the more robust the algorithm-determined starting point is, although more data will be required while a larger $K$ value will also lead to the delay of the starting point. In contrast, the smaller the value of $K$ is, the greater the sensitivity of the calculation is, although misjudgments may arise.

\subsection{Adaptive recognition for the end of the predictive algorithm}

This study focuses on the actual applicability of the algorithm in small embedded systems used in real-time signal processing of 
intelligent moisture analyzers. As such, systems require faster data-processing speeds and better real-time performance, the computational complexity of data fusion technology means it is not suitable for use in such circumstances. During the drying process, the mass of the milled rice sample was here measured every $6 \mathrm{~s}$ and the data uploaded to the computer. The set-up can be assumed as a single sensor and a slowly changing system. Taking the features of this system into consideration, the weighted average method and batch estimate method were then used to process weight data.

The permitted levels of moisture in milled rice according to Chinese standard GB 1354-2009 ${ }^{[26]}$ are shown in Table 3. As these data can be regarded as a priori knowledge of the level of milled rice moisture content, we set the first degree threshold value to $\delta_{1}=0.15$ (dry basis). $\quad \hat{M}_{\infty}^{(j)}$ is the $j_{\text {th }}$ predictive value of the sample. If $\hat{M}_{\infty}^{(j)} \geq \delta_{1}$, the value of $\hat{M}_{\infty}^{(j)}$ would be saved in an array $m 1[L]$, where $L$ is the length of the array. Calculated by experiment to select the best results, we set $L=20$.

Table 3 Permitted level of milled rice moisture

\begin{tabular}{lcc}
\hline \multicolumn{1}{c}{ Product name } & $\begin{array}{c}\text { water content } \\
\text { (wet basis) }\end{array}$ & $\begin{array}{c}\text { water content } \\
\text { (dry basis) }\end{array}$ \\
\hline long shaped rice & $\leq 14.5$ & $\leq 0.1696$ \\
round shaped rice & $\leq 15.5$ & $\leq 0.1834$ \\
long shaped glutinous rice & $\leq 14.5$ & $\leq 0.1696$ \\
round shaped glutinous rice & $\leq 15.5$ & $\leq 0.1834$ \\
\hline
\end{tabular}

Based on both theoretical analysis and experimental proof, during the accelerating and decelerating drying periods, the variation in the drying curve is rather different, which leads to relatively large errors in pre- and post-drying predicted moisture content. As a result, the Grubbs criterion is typically used to identify and remove invalid data ${ }^{[27]}$.

The data-weighted average of a predictive value is calculated by:

$$
\overline{\hat{M}}_{\infty}=\frac{1}{L} \sum_{j=1}^{L} \omega_{j} \hat{M}_{\infty}^{j}
$$

where, $\omega_{j}$ is the weight of the first group corresponding to the respective predictive value, and $L$ is the length of the array. The standard deviation can be calculated as:

$$
\sigma=\sqrt{\frac{1}{L-1} \sum_{j=1}^{L}\left(\hat{M}_{\infty}^{j}-\overline{\hat{M}}_{\infty}\right)^{2}}
$$

If one of the predictive value $\hat{M}_{e}^{j}$ residual errors $\left(E_{\mathrm{i}}\right)$ satisfies the formula:

$$
E_{i}=\left|\hat{M}_{\infty}^{j}-\overline{\hat{M}}_{\infty}\right| \geq g_{0}(n, \theta) \sigma
$$

Then $\hat{M}_{e}^{j}$ can be considered a bad value and is removed. In Equation (18), the term $g_{0}$ represents the Grubbs criterion discriminate coefficient, $n$ represents the measurement time, $\theta$ is the degree of significance and is generally either 0.05 or 0.01 , and $\sigma$ is the standard deviation ${ }^{[27]}$. The Grubbs criterion distinguishing method can be used repeatedly until no gross or fault errors are detected. Firstly, set $\delta_{1}$ as the first degree threshold value to determine whether the predicted value is close to the actual value. As the array length $L$, the second level threshold $\delta_{2}$ is a test parameter. Through a large number of experiments we set $\delta_{2}=0.002$ as the second degree threshold value to control the accuracy of the prediction and fusion algorithm. The predicted value in the array is then sorted according to ascending order. If the maximum and minimum values meet the criterion:

$$
\left|\hat{M}_{\max }-\overline{\hat{M}}_{\infty}^{(j)}\right| \leq \delta_{2} \mathrm{I}\left|\hat{M}_{\min }-\overline{\hat{M}}_{\infty}^{(j)}\right| \leq \delta_{2}
$$

then $\overline{\hat{M}}_{\infty}^{(j)}$ is the final predicted value of the algorithm. The following step-by-step methodology of drying model parameter identification is based on the LM algorithm and adaptive decision-making regarding the starting point and termination criteria. Its flowchart is shown in Figure 5.

Step 1: Calculate the rate of water loss and the first order derivative during the drying process;

Step 2: As illustrated in Equation (12), if $i+1$ meets the requirement of $S=1$, and the subsequent six points all meet the requirement, then $i+1$ is determined as the starting point of predictive algorithm;

Step 3: The sliding window sampling method is applied to the prediction algorithm, using a sliding data window array with length of 20. The predicted value of sample final moisture content (dry basis) $\hat{M}_{\infty}^{(j)}$ can then be calculated based on experimental data;

Step 4: Choose an initial set of parameters $\bar{k}=\left[M_{0}, M_{e}, \alpha, \beta\right]$ and an initial value of the damping parameter (here $\lambda_{0}=0.01$ ). The iteration number is then initialized $(j=0)$;

Step 5: Compute $\psi(\bar{k})$ from Equation (3);

Step 6: Compute the Jacobian matrix $\nabla \psi(\bar{k})$ defined by Equation (7) and the new set of estimates $\bar{k}_{j+1}$ from Equation (5), in which the unit matrix is replaced by the diagonal of the Hessian matrix;

Step7): Solve the direct problem using the new estimate $\bar{k}_{j+1}$ in order to find $f\left(x_{i}, \bar{k}_{j+1}\right)$. Then compute $\psi\left(\bar{k}_{j+1}\right)$, as defined in step 5. If $\psi\left(\bar{k}_{j+1}\right) \geq \psi\left(\bar{k}_{j}\right)$, replace $\lambda_{j}$ with $\lambda_{j+1}=10 \lambda_{j}$ and return to step 5. If $\psi\left(\bar{k}_{j+1}\right) \leq \psi\left(\bar{k}_{j}\right)$, accept the new set of estimates $\bar{k}_{j+1}$ and replace $\lambda_{j}$ with $\lambda_{j+1}=0.1 \lambda_{j}$. Check the two levels threshold criteria and stop the iterative procedure if satisfying.

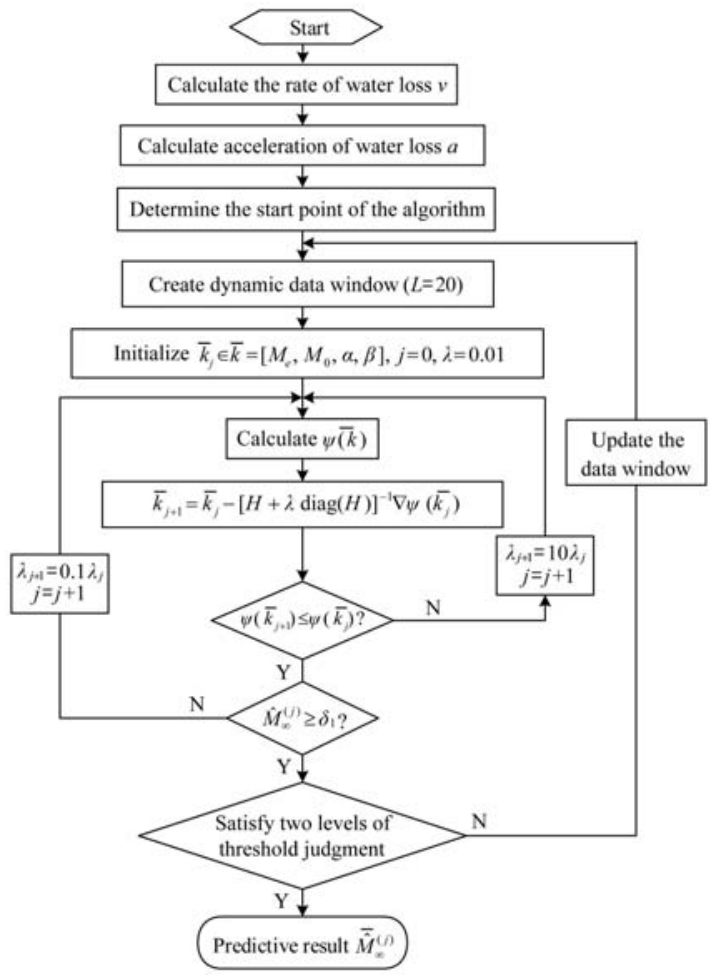

Figure 5 Flow chart of the adaptive LM predictive algorithm 


\subsection{Experimental identification of the algorithm}

Table 4 compares the algorithm-predicted results with the observed results obtained via the AOAC loss on drying method at $105^{\circ} \mathrm{C}$. Analysis of these data reveals good agreement between the measured and predicted moisture content values. For milled rice samples varying in moisture content from $14.44 \%-1767 \%$ (dry basis), the relative error between the predicted and observed values was $0.0037-0.0589$, with a reduction in test time of about $50.71 \%$ $67.87 \%$.

Table 4 Comparison of predicted and experimental values of milled rice moisture content

\begin{tabular}{cccccccc}
\hline No. & $\begin{array}{c}\text { Mass } \\
\text { /g }\end{array}$ & $\begin{array}{c}\text { Observed } \\
\text { value } \\
\text { /\%(d.b.) }\end{array}$ & $\begin{array}{c}\text { Predicted } \\
\text { value } \\
\text { /\%(d.b.) }\end{array}$ & $\begin{array}{c}\text { Relative } \\
\text { error/\% }\end{array}$ & $\begin{array}{c}\text { Measured } \\
\text { time } \\
/ \text { min }\end{array}$ & $\begin{array}{c}\text { Predicted } \\
\text { time } \\
/ \text { min }\end{array}$ & $\begin{array}{c}\text { Time } \\
\text { saving } \\
/ \%\end{array}$ \\
\hline 1 & 4.991 & 11.17 & 11.51 & 0.34 & 33.60 & 16.40 & 51.19 \\
2 & 5.615 & 11.56 & 11.94 & 0.38 & 33.90 & 16.50 & 51.33 \\
3 & 4.983 & 13.55 & 13.83 & 0.28 & 37.90 & 19.20 & 49.34 \\
4 & 4.009 & 11.96 & 12.56 & 0.60 & 30.20 & 16.90 & 44.04 \\
5 & 5.180 & 12.31 & 12.05 & 0.26 & 39.90 & 18.90 & 52.63 \\
6 & 5.324 & 12.01 & 11.92 & 0.09 & 39.50 & 18.30 & 53.67 \\
7 & 4.768 & 11.02 & 11.31 & 0.29 & 33.70 & 16.50 & 51.04 \\
8 & 6.502 & 12.35 & 12.13 & 0.22 & 40.60 & 17.30 & 57.39 \\
9 & 5.556 & 11.92 & 11.74 & 0.18 & 29.30 & 14.10 & 51.88 \\
10 & 4.998 & 13.07 & 13.83 & 0.76 & 41.20 & 16.70 & 59.47 \\
11 & 4.997 & 12.88 & 12.23 & 0.65 & 40.20 & 16.50 & 58.96 \\
12 & 6.182 & 12.04 & 12.57 & 0.53 & 39.90 & 19.90 & 50.12 \\
13 & 7.502 & 12.81 & 12.92 & 0.11 & 42.50 & 20.50 & 51.76 \\
14 & 8.500 & 12.15 & 12.33 & 0.18 & 38.70 & 16.70 & 56.85 \\
15 & 9.997 & 11.97 & 11.42 & 0.55 & 33.60 & 15.20 & 54.76 \\
\hline & & & & & & &
\end{tabular}

\section{Conclusions}

The investigation of this study has shown that the numerical model based on the Weibull distribution is suitable for predicting the moisture content of milled rice. This research also extended and generalized the approach by further developing the information fusion method for the prediction of moisture content using the LM method, with the predictive results then compared with data obtained via $\mathrm{AOAC}$ oven drying at $105^{\circ} \mathrm{C}$. For milled rice samples varying in moisture content from $14.44 \%-17.67 \%$ (dry basis), the relative error between the predicted and observed values was $0.0037-0.0589$, with a reduction in test time of about $50.71 \%-67.87 \%$. The research results may be beneficial in finding an effective method suitable for small embedded systems used in the real-time signal processing of predictive moisture analyzers.

\section{Acknowledgements}

This study supported by a grant from the National Natural Science Foundation of China (No.61663039), Natural Science Foundation of Ningxia Hui Autonomous Region (No. NZ1648) and the Natural Science Funds of Ningxia University (ZR15010).

\section{[References]}

[1] Cihan A, Kahveci K, Hacihafizoglu O. Modelling of intermittent drying of thin layer rough rice. Journal of Food Engineering, 2007; 79(1): 293-298.

[2] Lopes D D C, Neto A J S, Santiago J K. Comparison of equilibrium and logarithmic models for grain drying. Biosystems Engineering, 2014; 118(1): 105-114

[3] AOAC. Moisture in cereal adjuncts: Air oven method $\left(103\right.$ to $\left.104^{\circ} \mathrm{C}\right)$.
Official Methods of Analysis. 15th ed. Arlington, VA: Association of Official Analytical Chemists, 1990.

[4] International Organization for Standardization (ISO) 712. Cereals and cereal products - Determination of moisture content (Routine reference method). Geneva, Switzerland: International Standard ISO, 1998.

[5] Nelson S O, Trabelsi S. Historical development of grain moisture measurement and other food quality sensing through electrical properties. IEEE Instrumentation \& Measurement Magazine, 2016; 19(1): 16-23.

[6] Nath K D, Das Y M S, Ramasamy S, Ramanathan P. A review on non-destructive methods for the measurement of moisture contents in food items// International Conference on Circuit, Power and Computing Technologies. IEEE, 2015: 1-6.

[7] Borompichaichartkul C, Moran G, Srzednicki G, Price W S. Nuclear magnetic resonance (NMR) and magnetic resonance imaging (MRI) studies of corn at subzero temperatures. Journal of Food Engineering, 2016; 69(2): 199-205.

[8] Knegt R J D, Brink H V D. Improvement of the drying oven method for the determination of the moisture content of milk powder. International Dairy Journal, 1998; 8(8): 733-738.

[9] Baysal T, Icier F, Ersus S, Yildiz H. Effects of microwave and infrared drying on the quality of carrot and garlic. European Food Research \& Technology, 2003; 218(1): 68-73.

[10] Novak J F, Burch M R. Method and apparatus for microwave utilization: US, US6618957, 2003.

[11] Shrivastav S, Kumbhar B K. Modeling and optimization for prediction of moisture content, drying rates and moisture ratio. Int J Agric \& Biol Eng, 2009; 2(1): 58-64.

[12] Ling J, Teng Z S, Lin H J, Zhang K X. Estimation and fusion model for meat moisture content measurement based on loss-on-drying. Chinese Journal of Scientific Instrument, 2015; 36(2): 318-326. (in Chinese)

[13] Corzo O, Bracho N, Pereira A, Alberto V. Weibull distribution for modeling air drying of coroba slices. LWT-Food Science and Technology, 2008; 41(10): 2023-2028.

[14] Models MA100/MA50 electronic moisture analyzer operating instructions. Sartorius, Beijing, 2004

[15] MA50 and MA100 user's manual. Sartorius, Beijing, 2004.

[16] Ling J, Teng Z S, Lin H J, Wen H. Infrared drying kinetics and moisture diffusivity modeling of pork. Int J Agric \& Biol Eng, 2017; 10(3): 302-311.

[17] Corzo O, Bracho N, Rodriacute J. Application of Weibull distribution model in describing the pile salting of goat meat slices. 2013; 8(14): $581-588$

[18] Sant'Anna V, Utpott M, Claderaolivera F, Brandelli A. Kinetic modeling of the thermal inactivation of Bacteriocin-like inhibitory substance P34. Journal of Agricultural \& Food Chemistry, 2010; 58(5): 3147-3152.

[19] Marabi A, Saguy I S. Viscosity and starch particle size effects on rehydration of freeze-dried carrots. Journal of the Science of Food \& Agriculture, 2005; 85(4): 700-706.

[20] Corzo O, Bracho N, Alvarez C. Weibull model for thin-layer drying of mango slices at different maturity stages. Journal of Food Processing \& Preservation, 2010; 34(6): 993-1008.

[21] Cunha L M, Oliveira F A R, Oliveira J C. Optimal experimental design for estimating the kinetic parameters of processes described by the Weibull probability distribution function. Journal of Food Engineering, 1998; 37(2): 175-191.

[22] Rouquette S, Guo J, Masson P L. Estimation of the parameters of a Gaussian heat source by the Levenberg-Marquardt method: Application to the electron beam welding. International Journal of Thermal Sciences, 2007; 46(2): 128-138.

[23] Marquardt D W. An algorithm for least-squares estimation of nonlinear parameters. Journal of the Society for Industrial and Applied Mathematics, 1963; 11(2): 431-441.

[24] Le D H, Cong-Kha P, Trang N T Tu B T. Parameter extraction and optimization using Levenberg-Marquardt algorithm. Fourth International Conference on Communications and Electronics (ICCE), IEEE, 2012; 434-437.

[25] Ahmed J, Al-Attar H. Effect of drying method on rheological, thermal, and structural properties of chestnut flour doughs. Food Hydrocolloids, 2015; 51: 76-87.

[26] Chinese National standards. GB11354-2009 Grain. Beijing: Standard Press of China, 2009. (in Chinese)

[27] Shi Y, Luo Y, Zhao W, Shang C, Wang Y. A radiosonde using a humidity sensor array with a platinum resistance heater and multi-sensor data fusion. Sensors, 2013; 13(7): 8977-8996. 